JOURNAL OF

SYMPLECTIC GEOMETRY

Volume 11, Number 2, 317-318, 2013

\title{
CORRIGENDUM TO \\ SCALING LIMITS FOR EQUIVARIANT SZEGÖ KERNELS
}

\author{
Roberto PaOletti
}

A computational error involving an index occurred in the course of the proof of Theorem 1, affecting the displayed formula in the Theorem. More precisely, the last two displayed formulae on page 28 should read

$$
\psi_{2}\left(w_{j}-\nu_{M}(m), v\right)+\mathrm{i} \omega_{m}\left(\nu_{M}(m), w_{j}\right)=T_{\mathrm{h}}^{(j)}+T_{\mathrm{t}}^{(j)}+T_{\mathrm{v}}^{(j)}+T_{\mathrm{vt}}^{(j)},
$$

and

$$
\begin{aligned}
& T_{\mathrm{h}}^{(j)}=: \psi_{2}\left(w_{j \mathrm{~h}}, v_{\mathrm{h}}\right), T_{\mathrm{t}}^{(j)}=:-\frac{1}{2}\left\|w_{j \mathrm{t}}-v_{\mathrm{t}}\right\|^{2}, \\
& T_{\mathrm{v}}^{(j)}=:-\frac{1}{2}\left\|w_{j \mathrm{v}}-\nu_{M}(m)-v_{\mathrm{v}}\right\|^{2}, \\
& T_{\mathrm{tv}}^{(j)}=:-i \omega_{m}\left(w_{j \mathrm{v}}-\nu_{M}(m)-v_{\mathrm{v}}, w_{j \mathrm{t}}+v_{\mathrm{t}}\right)+\mathrm{i}\left[\omega_{m}\left(w_{\mathrm{v}}, w_{\mathrm{t}}\right)-\omega_{m}\left(v_{\mathrm{v}}, v_{\mathrm{t}}\right)\right],
\end{aligned}
$$

where the unitary endomorphism $w \mapsto w_{j}$ of $T_{m} M$ is induced by the isotropy action of $g_{j}^{-1} \in G_{m} \subseteq G$. This implies that in the exponent appearing in formula (52) the factor $\Gamma(w, v)$ must be replaced by

$$
\Gamma\left(w_{j}, v\right)=\psi_{2}\left(w_{j \mathrm{~h}}, v_{\mathrm{h}}\right)-\left\|w_{\mathrm{t}}\right\|^{2}-\left\|v_{\mathrm{t}}\right\|^{2}+\mathrm{i}\left[\omega_{m}\left(w_{\mathrm{v}}, w_{\mathrm{t}}\right)-\omega_{m}\left(v_{\mathrm{v}}, v_{\mathrm{t}}\right)\right] .
$$

In the notation of the paper, let us set

$$
A_{\varpi, k}(g, x)=: 2^{\mathrm{g} / 2} \frac{\operatorname{dim}\left(V_{\varpi}\right)}{V_{\mathrm{eff}}(x)} \cdot \frac{1}{\left|G_{\pi(x)}\right|} \chi_{\varpi}(g) h_{g}^{k} \quad\left(g \in G_{\pi(x)}\right) .
$$


With the rest of the argument unchanged, the outcome is that in Theorem 1 the displayed formula should read:

$$
\begin{aligned}
\Pi_{\varpi, k} & \left(x+\frac{w}{\sqrt{k}}, x+\frac{v}{\sqrt{k}}\right) \\
& \sim\left(\frac{k}{\pi}\right)^{\mathrm{n}-\mathrm{g} / 2} \mathrm{e}^{Q\left(w_{\mathrm{v}}+w_{\mathrm{t}}, v_{\mathrm{v}}+v_{\mathrm{t}}\right)} \sum_{g \in G_{m}} A_{\varpi, k}(g, x) \mathrm{e}^{\psi\left(w_{g \mathrm{~h}}, v_{\mathrm{h}}\right)} \\
& \times\left(1+\sum_{j \geq 1} a_{\varpi j}\left(x, w_{g}, v\right) k^{-j / 2}\right)
\end{aligned}
$$

where $w \mapsto w_{g}$ is the isotropy action of $g \in G_{m}$, and just as before

$$
Q\left(w_{\mathrm{v}}+w_{\mathrm{t}}, v_{\mathrm{v}}+v_{\mathrm{t}}\right)=-\left\|v_{\mathrm{t}}\right\|^{2}-\left\|w_{\mathrm{t}}\right\|^{2}+\mathrm{i}\left[\omega_{m}\left(w_{\mathrm{v}}, w_{\mathrm{t}}\right)-\omega_{m}\left(v_{\mathrm{v}}, v_{\mathrm{t}}\right)\right] .
$$

Dipartimento di Matematica e Applicazioni

Università degli Studi di Milano Bicocca

ViA R. Cozzi 53

20125 Milano

ITALY

E-mail address: roberto.paoletti@unimib.it

Received 11/21/2012, accepted 01/07/2013 